\title{
Image Denoising With Improved Switching Median Mean Filter Using Level Shifting
}

\author{
Sukhninder Singh ${ }^{1}$, Mrs Neetu ${ }^{2}$ \\ ${ }^{1,2}$ (Department of ECE, Beant College of Engineering and Technology, Gurdaspur, Punjab, India)
}

\begin{abstract}
Digital Image Processing involves the variation of digital data for improving the qualities of image with the support of computer.The use of decision based trimming of noisy pixels is ignored in the existing literature. Also the effects of different kind of noises are also ignored. The use of level shifting for correcting the borders and preserving the edges are also not considered. Recently much work is done in the different fields like medical, remote sensing, military applications etc. to improve the visibility of digital images. Many algorithms have been proposed so far for enhancing the digital images. It has been found that the most of the existing researchers have neglected many issues; i.e. no technique is accurate for different kind of circumstances.
\end{abstract}

Keywords: Digital Image Processing, types of noises, File Formats

\section{Introduction}

Image processing allocates with function of electronic picture via electronic computer. It targeted usually on photos but in addition subfield of signals and systems. It targeted on handling a computer program that is able to apply handling on a picture. The feedback to facilitate program is just a digita1 picture and the machine process to facilitate picture using well-ordered programs, and provides a picture as outcome. Adobe Photoshop is the common example. It's among the largely used applications for handling on electronic photos [1].

Images are the most frequent and easy way of transferring or sending information. A picture is worth a plenty of words. Images concisely share details about positions, dimensions and inter-relationships between objects. They portray spatial data that we can recognize as objects. People are good at deriving data from such photos, due to our innate visual and mental abilities. About $75 \%$ of the info received by individual is in pictorial form. In the current situation, the analyses of pictures that employ an expense perspective, such as the radiation perhaps not apparent to human eye are considered.

Hence our discussion will soon be directing on analysis of slightly sensed images. These photos are represented in electronic form. When represented as numbers, perfection can be included, deduced, increased, split and, generally, afflicted by statistical manipulations that aren't possible if a picture is presented just as a photograph. Though electronic analysis of slightly sensed knowledge times from the first times of rural sensing, the release of the first Land sat planet remark satellite in 1972 began a time of raising curiosity about device handling (Cambell, 1996 and Jensen, 1996). Formerly, electronic rural sensing knowledge might be reviewed just at specialized rural sensing laboratories. Particular gear and qualified personnel necessary to perform routine device analysis of knowledge were not generally available, in part due to confined option of electronic rural sensing knowledge and a lack of understanding of their qualities. A digital slightly sensed picture is usually made up of image components (pixels) based at the intersection of each row $\mathrm{i}$ and column $\mathrm{n}$ in each $\mathrm{E}$ artists of imagery. Related to each pixel is a Digital Number (DN) or Brightness Value (BV) that depicts the typical radiance of a somewhat little place.

Digital image might be broken by various tones in certain applications. Attenuation noise is a vital task in digital image processing. The problem of this work is how to lessen while maintain the image detail. There are several works on repair images broken by intuition noise. Most popular filtration is median filter; however it smears some facts and ends of unique images particularly once the noise stage is high [2]. In this paper, Improved Switching Median Mean filter is used for de-noising the image.

\section{Types Of Noises}

There are various types of noises which get added in digital images.

\subsection{Impulse Noise (Salt and Pepper Noise)}

The definition of intuition noise can be employed for this sort of sound [2]. Different phrases are spike noise, random noise or independent noise. Dark and white spots appear in the picture [5] consequently of this sound and ergo salt and pepper noise. This noise arises in the picture due to sharp and unexpected improvements of picture signal. Dirt particles in the picture exchange source or higher hot bad components may cause this sort of noise. Picture is corrupted to a tiny degree due to noise. 


\subsection{Gaussian Noise (Amplifier Noise)}

The definition of normal sound model may be the synonym of Gaussian noise. This noise model is additive in nature [4] and follow Gaussian distribution. Meaning that each pixel in the loud picture may be the amount of the true pixel value and a random, Gaussian distributed sound value. The sound is independent of power of pixel value at each point.

\subsection{Uniform Noise}

The uniform noise cause by quantizing the pixels of picture to a number of distinct degrees is known as quantization noise. It has approximately uniform distribution. In the uniform sound the amount of the gray prices of the sound are uniformly distributed across a given range. Standard sound can be used to make any various form of sound distribution.

\subsection{Gamma Noise}

The sound could be obtained by the low-pass filtering of laser based images.

\section{File Formats}

There are numerous audio types, there are numerous image types, and in this part we can give shallow information of a number of them. The record format is critical to the preservation of an image. The TIFF record (tagged image record format) is the present preservation format. This is because it holds all the spatial (resolution), color, tone, metadata and preservation information needed to create a digital master of the first;

Gif. Graphics Interchange Format was introduced in 1987 as a concise illustration of color images. It runs on the scheme for the most part 256 colors sampled from the 24-bit color model. What this means is that it's unsuitable for color images with continuous color tones, but it works very well for smaller images with large aspects of continuous color, like logos and links on internet pages. Gif-files squeezed by using the Lempel-ZivWelch algorithm to lessen the file size without degrading the visible quality. The extension of GIF-files is .gif.

Tiff. Tagged Image File Format is just a variable image format that may contain multiple images of different kinds in the same record via alleged labels. TIFF supports lossless image pressure via Lempel-ZivWelch pressure, but may also contain JPEG-compressed images. TIFF was formerly developed as a structure for scanned documents and supports images with one-bit pixel values (black and white). It also supports sophisticated data types like a lot more than ten portions per color component. TIFF-files have extension .tiff.

Jpeg. Joint Photographic Experts Group is a picture format which was permitted being an international common in 1994. JPEG is normally lossy, but may also be lossless and has turned into a common format for image illustration on the Internet. The typical defines both the algorithms for encoding and decoding and the storage format. JPEG divides the image into $8 \times 8$ prevents and changes each block with a Distinct Cosine Transform. These values similar to higher 359 wavelengths (rapid variations in color) are then collection to 0 unless they're really large, as this is not noticed much by human perception. The perturbed DCT values are then numbered by an alternative of Huffman coding. JPEG may also use arithmetic code, but this increases both the encoding and decoding times, with no more than $5 \%$ development in the pressure ratio. The pressure level in JPEG images is selected by the consumer and may bring about conspicuous artifacts if collection too high. JPEG is particularly susceptible to artifacts in areas where in fact the intensity changes quickly from pixel to pixel. The extension of a JPEG-file is .jpg or .jpeg.

Png. Portable Network Graphics is just a lossless image format which was printed in 1996. PNG was not designed for skilled use, but rather for transferring images on the Web, and just supports grey-level images and RGB images (also scheme based color images). PNG was made to prevent a patent on the LZW-algorithm used in GIF, and also GIF's limitation to ten bit color information. For effective code PNG may (this is an option) estimate the value of a pixel from the value of prior pixels, and subtract the believed price from the actual value. It can then rule these mistake values using a lossless code technique called DEFLATE which employs a variety of the LZ77 algorithm and Huffman coding. This is similar to the algorithm used in lossless audio types like Apple Lossless and FLAC. The extension of PNG-files is .png.

Jpeg 2000. This lossy (can also be properly used as lossless) image format was developed by the Mutual Final Specialists Group and printed in 2000. JPEG 2000 changes the image data with a wavelet convert rather than a DCT. Following substantial control of the wavelet coefficients, the last code runs on the version of arithmetic coding. At the cost of improved encoding and decoding times, JPEG 2000 contributes to around $20 \%$ development in pressure ratios for moderate pressure charges, probably more for large or minimal pressure rates. The artifacts are less visible than in JPEG and look at larger pressure rates. While several components in JPEG 2000 are patent, the patent members have agreed that the core computer software must be around totally free, and JPEG 2000 is part of most Linux distributions. Nevertheless, there appear to be some further, rather hidden, patents that have maybe not been registered, and this might be exactly why JPEG 2000 isn't applied more. The extension of JPEG 2000 documents is .jp2. 


\section{Literature Review}

Chengbin Zhang et al.2015 [1] defined a brand new algorithm to remove intuition noise from highly broken images. This technique can not just achieve better image quality, but also have smaller computation time. And this approach is simple and easy to be understood. Simulation results reveal that this approach provides better performance than the existing strategy presented for broken by highly noise thickness in terms of noise reduction and detail preservation. This technique provides an successful filter body and is ideal for real time implementation because it works on the set $3 \times 3$ window measurement for filtering processing.

Priyanka Kamboj et al. 2013 [2] attracted the general picture of numerous noise models in addition to various filtration techniques. Through the duration of image handling, noise reduction along with repair of the image is supposed to enhance the qualitative examination associated by having an image and the specific performance conditions connected with quantitative image examination methods Electronic images is inclined to numerous tones which influence the typical of image. The main goal of de-noising the image is usually to displace the facet of original image when possible. They learned filters such as linear, non-linear filters in addition to cross filter system (in which two or more filters are used).In the area of lowering the specific image noise a few kind of linear and non-linear filter methods are now proposed.

Yang, Pan et al. 2013 [3] proposed a data obfuscation system that gives a defined "noise" to the original data to shield the privacy while keeping the numeral features of data unaffected in several levels. Besides, the system may also lower the affect on data mining. Furthermore, by allocating various tips to customers, various customers have various permissions to find you in to data. To create this happen, this system will be four steps. Firstly, a larger cloud product is proposed to create a defined "noise ".Next, an obfuscation algorithm is proposed to include noise to the original data. In the best step, a fine-grained collection system based on likeness is proposed. The experiments indicated that their system obfuscates time correctly, successfully, and securely.

Jian Zhanga et al. 2015 [4] proposed an algorithm for image de-noising can only look after a single sort of sound, which violates the opinion that the loud seen photos used in many cases are endured many type of sound through the procedure of order and transmission. Additionally, a Split-Bregman based process is created to solve the above optimization issue efficiently. Intensive experiments for blended Gaussian moreover wish sound reveal that substantial performance changes on the existing state-of-the-art schemes already are accomplished, which substantiates the potency of the proposed formula.

Xuechen Li et al. 2012 [5] showed some experiments along with algorithm to be able to curb the wish sound inside the image of one's welded joint correctly, the increased changing median (ISM) selection is proposed. In the algorithm, the neighborhood studies based sound sensor may be adopted to categorize the pixels inside the image in to uncorrupted pixels along with sound pixels. The particular noise-free pixels are often remaining unprocessed. Experimental effects display that ISM filtration may curb sound correctly while preserving great facts adequately. To be able to overcome the disadvantage of TM filters the various changing based filters are proposed. The ISM filtration gains the well-known gain around your changing median (SM) selection, filtration based about serious and normal value (EM) along with progressive changing median (PSM) selection, especially when your density sound inside the image is fairly high.

Sachin et al. 2012 [6] interviewed the true record and purposes of data mining practices in the educational field. The objective is always to introduce data mining to standard educational process, web-based educational process, clever tutoring process, and e-learning. They defined how to utilize the main data mining practices such as for example prediction, classification, connection mining, clustering, and cultural area network to educational data.

Kamishima et al. 2012 [7] investigated the formal ideas of fairness and techniques for handling fairness in data mining. As knowledge mining is simply a strong new engineering which concentrate on the most vital data present in the data. Then they offered to see of those ideas dedicated to mathematical independence. Finally, they mentioned the relations between fairness-aware data mining and different study subjects, such as for example privacy-preserving data mining or causal inference.

Cao Longbing et al. 2012 [8] drew a comprehensive overall image and summarizes the equivalent practices and cases to analyze cultural security/welfare knowledge, particularly, cultural security data mining (SSDM), dedicated to an intensive overview of a considerable number of related references from days gone by half century. Especially, they introduced an SSDM construction, including business and study dilemmas, cultural security/welfare companies and knowledge, along with challenges, goals, and responsibilities in mining cultural security/welfare data.

Farid et al. 2012 [9] planned a whole new strategy for finding novel class in data stream mining applying decision tree classifier which will determine whether an hidden or new example belongs to an account class or not. Most existing data mining classifiers cannot discover and identify the novel class cases in real-time data stream mining problems like temperature conditions, economical improvements, astronomical, and intrusion recognition an such like, before the classification versions are trained with the 
labeled cases of the novel class. Birth of an account class in concept-drift happens in data stream mining when new data present the most recent idea classes or eliminate the previous ones. The experiments on real benchmark data consider the performance of the planned strategy in equally finding the class and classification precision with comparisons of traditional data mining classifiers.

Madraky et al. 2012 [10] planned an intermediate data product that might represented perfect for Spatiotemporal data and performing data mining task quickly while facing problem in usually adjusting the data. In order to propose suitable data product, that study also investigate the diagnostic parameters, the structure and their specifications for spatiotemporal data. The notion of proposed knowledge product is influenced from the type of hair that's certain attributes and their development within the time. In order to have greater seeking and quality, the data is necessary to keep over the time such as for example as an example combing, cutting, coloring, covering, washing etc. The proposed data product is represented by using mathematical product and later developed the data product tools. The data product is developed based on the current relational and objectoriented models.

Kostadin Dabov et al. 2006 [11] presented a novel way of still image de-noising based on effective filtering in 3D change domain by mixing sliding-window change handling with block-matching. We method prevents within the image in a falling fashion and utilize the block-matching concept by searching for prevents which are just like the presently prepared one. The matched prevents are piled together to make a 3D range and as a result of likeness between them, the information in the range show higher level of correlation. We exploit this relationship by making use of a 3D de-correlating unitary change and effectively attenuate the noise by shrinkage of the change coefficients. The following inverse 3D change produces estimates of most matched blocks. After saying this process of all images prevents in falling fashion, the last estimate is computed as considered normal of most overlapping block estimates. A quick and efficient algorithm employing the proposed method is developed. The fresh benefits show that the proposed method produces state-of-art denoising performance, equally in terms of goal standards and visual quality.

Yang, Pan et al. 2013 [12] planned a data obfuscation scheme that gives an exact "noise" to the original data to guard the solitude while keeping the numeral features of data unaffected in a variety of levels. Besides, the scheme can also decrease the influence on data mining. Furthermore, by allocating different recommendations to people, different people have different permissions to get involved with to data. To create that happen, that scheme comes into play four steps. Firstly, a larger cloud product is planned to produce an exact "noise ".Next, an obfuscation algorithm is planned to add noise to the original data. In the best step, a finegrained bunch scheme based on likeness is proposed. The experiments suggested that their scheme obfuscates day properly, efficiently, and securely.

V. Results And Discussions

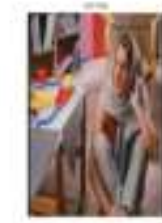

a)Input Image1

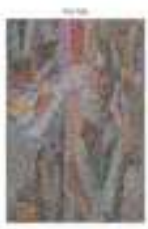

b) Noisy Lnage

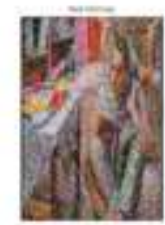

e) Median Filter

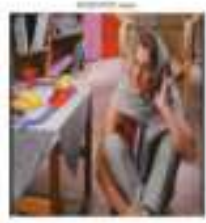

d) Basepaper Resalts

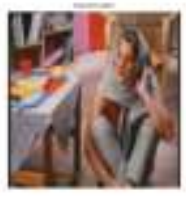

e) Proposed Results

Fig 1: Experimental Results of Image 1

The experimental results are taken under MATLAB version 2013. Firstly results are taken at noise density 0.6 in fig 1.

a) Input Image - Any color input image is taken for experimental purposes.

b) Noisy Image - Now the 0.6 noise density is added in the input image.

c) Median Filter Image - This result shows the output of median filter.

d) Base-paper Results - This result shows the output of existing algorithm.

e) Proposed Results - This result shows the output of proposed algorithm.

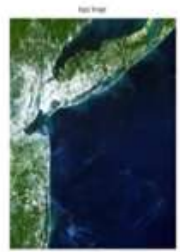

a)Input Image 3

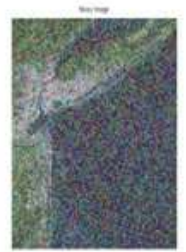

b) Noisy Image

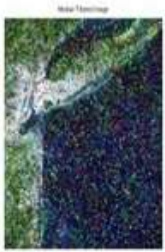

c) Median Filter

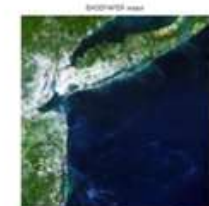

d) Basepaper Results

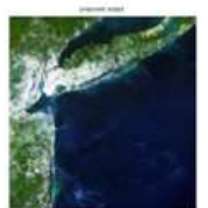

e) Proposed Results

Fig 2: Experimental Results of Image 3 


\section{Performance Analysis}

6.1 MSE --Mean square error is to compute an error signal by subtracting the test signal from the reference, and then computing the average energy of the error signal. It can be explained as:

$$
\operatorname{MSE}=\frac{1}{M N} \sum_{i=1}^{M} \sum_{j=1}^{N}\left(f(i, j)-f^{\prime}(i, j)\right)^{2}
$$

Table 1: MSE Comparison Table

\begin{tabular}{|l|l|l|l|l|}
\hline Input Images & Noisy Images & Median Filter & Basepaper Results & Proposed Results \\
\hline Input image1 & 11524 & 3976 & 409 & 274 \\
\hline Input image2 & 11035 & 4248 & 677 & 671 \\
\hline Input image3 & 15708 & 5510 & 551 & 462 \\
\hline Input image4 & 14594 & 4964 & 366 & 241 \\
\hline Input image5 & 14189 & 5159 & 967 & 747 \\
\hline Input image6 & 12767 & 4676 & 585 & 522 \\
\hline
\end{tabular}

Table 1 shows the MSE comparison results of noisy image, median filter image, base paper results and proposed results.

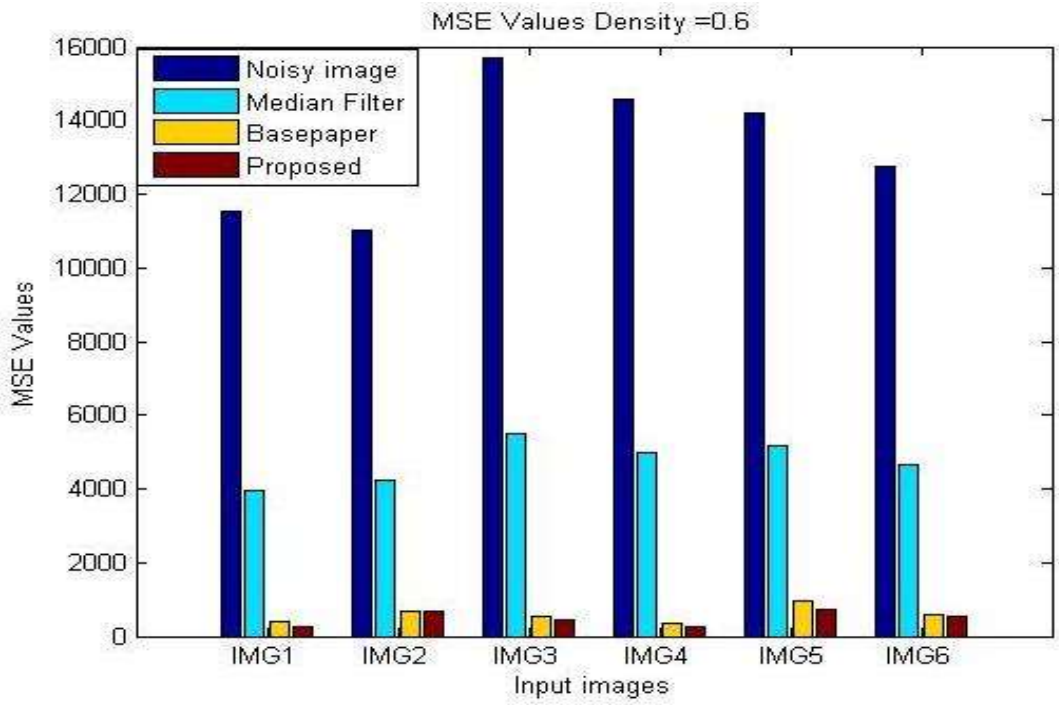

Fig 3: MSE Graph

Fig 3 shows that proposed are much better (i.e. low) than the existing results.

6.2 PSNR - Peak signal to noise ratio is the ratio between the maximum possible value of the signal and the power of the corrupting noise. It is measured in decibels $(\mathrm{db})$. It can be explained as:

PSNR $=10 \cdot \log _{10}\left(\frac{M A X_{I}^{2}}{M S E}\right)$

Table 2: PSNR Comparison Table

\begin{tabular}{|l|l|l|l|l|}
\hline Input Images & Noisy Images & Median Filter & Basepaper Results & Proposed Results \\
\hline Input image1 & 7.5148 & 12.1363 & 22.0136 & 23.7533 \\
\hline Input image2 & 7.7031 & 11.8490 & 19.8249 & 19.9121 \\
\hline Input image3 & 6.1696 & 10.7193 & 20.7193 & 21.4844 \\
\hline Input image4 & 6.4891 & 11.1725 & 22.4960 & 24.3106 \\
\hline Input image5 & 6.6113 & 11.0051 & 18.2765 & 19.3976 \\
\hline Input image6 & 7.0699 & 11.4321 & 20.4592 & 20.9541 \\
\hline
\end{tabular}

Table 2 shows the PSNR comparison results of noisy image, median filter image, base paper results and proposed results. 


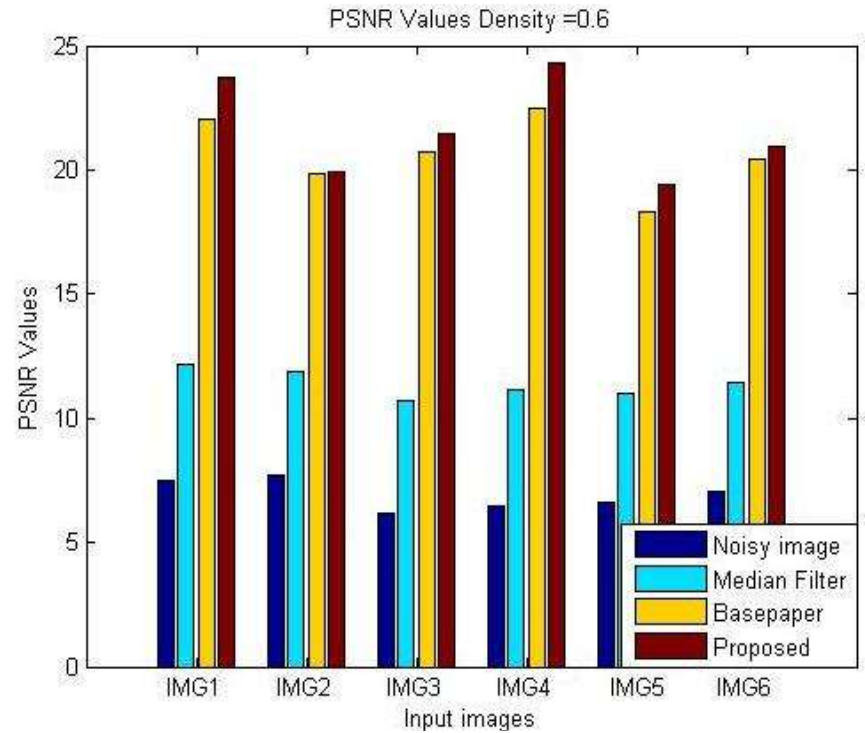

Fig 4: PSNR Graph

Fig. 4 depicts that proposed are much better (i.e. high) than the existing results.

6.3 BER - It is defined as the rate at which errors occur in a transmission system. This can be directly translated into the number of errors that occur in a string of a stated number of bits. The definition of bit error rate can be translated into a simple formula:

$$
B E R=\frac{\text { Number of errors }}{\text { Total number of bits sent }}
$$

Table 3: BER Comparison Table

\begin{tabular}{|l|l|l|l|l|}
\hline Input Images & Noisy Images & Median Filter & Basepaper Results & Proposed Results \\
\hline Input image1 & 0.1331 & 0.0824 & 0.0454 & 0.0421 \\
\hline Input image2 & 0.1298 & 0.0844 & 0.0504 & 0.0450 \\
\hline Input image3 & 0.1621 & 0.0933 & 0.0483 & 0.0465 \\
\hline Input image4 & 0.1541 & 0.0895 & 0.0445 & 0.0411 \\
\hline Input image5 & 0.1513 & 0.0909 & 0.0547 & 0.0516 \\
\hline Input image6 & 0.1414 & 0.0875 & 0.0489 & 0.0477 \\
\hline
\end{tabular}

Table 3 shows the MSE comparison results of noisy image, median filter image, base paper results and proposed results.

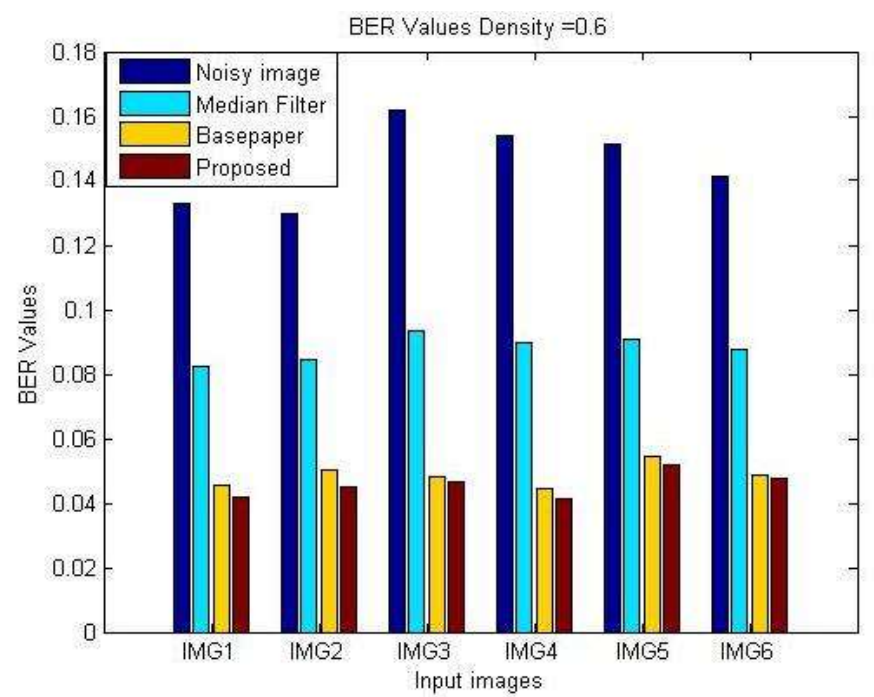

Fig 5: BER Graph

Fig. 5 depicts that proposed are much better (i.e. low) than the existing results. 


\section{Conclusion}

This specific work possesses a research on numerous filter techniques. The review has demonstrated that the particular still several improvements are usually require inside the available approaches to handle different type of images. This work has revealed that the particular no technique is beneficial for every type of images or perhaps images information set.To design and evaluate the performance of some well-known variants of median filters. To propose a new algorithm that will use decision based trimming strategy to find the best replacement for the noisy pixels.To preserve the edges and borders will also be used. The comparison among proposed and existing median filter techniques will also be drawn by taking some image performance parameters like peak signal to noise ratio, mean square error, and bit error rate.

\section{References}

[1]. Chengbin Zhang Kaifu Wang, "A switching median-mean filter for removal of high density impulse noise from digital images," in Optik-Int Journal for light and electron optics(IJLEO-Vol. 126),2015.

[2]. Priyanka Kamboj et al., "A Brief Study of Various Noise Model and Filtering Techniques" Journal of Global Research in Computer Science REVIEW ARTICLE Volume 4, No. 4, April 2013.

[3]. Yang, Pan, XiaolinGui, Feng Tian, Jing Yao, and Jiancai Lin. "A Privacy-Preserving Data Obfuscation Scheme Used in Data Statistics and Data Mining." in High Performance Computing and Communications \& 2013 IEEE International Conference on Embedded and Ubiquitous Computing, 2013 IEEE 10th International Conference on, pp. 881-887. IEEE, 2013.

[4]. Jian Zhanga et al., "Exploiting Image Local and Non-Local Consistency for Mixed Gaussian-Impluse Noise Removal." Research gate 4 feb 2015

[5]. Xuechen Li et al., "Improved Switching Median Filter for Removing Impulse Noise from the Image of the Welded Joint" 5th International Congress on Image and Signal Processing 2012

[6]. Sachin, R. Barahate, and M. Shelake Vijay. "A survey and future vision of data mining in educational field." In Advanced Computing \& Communication Technologies, 2012 Second International Conference on, pp. 96-100. IEEE, 2012.

[7]. Kamishima, Toshihiro, ShotaroAkaho, Hideki Asoh, and Jun Sakuma. "Considerations on fairness-aware data mining." In Data Mining Workshops, 2012 IEEE 12th International Conference on, pp. 378-385. IEEE, 2012.

[8]. Cao, Longbing. "Social security and social welfare data mining: An Overview."Systems, Man, and Cybernetics, Part C: Applications and Reviews, IEEE Transactions on 42, no. 6: 837-853, 2012

[9]. Farid, DewanMd, and Chowdhury Mofizur Rahman. "Novel class detection in concept-drifting data stream mining employing decision tree." In Electrical \& Computer Engineering, 7th International Conference on, pp. 630-633. IEEE, 2012.

[10]. Madraky, Abbas, Zulaiha Ali Othman, and Abdul Razak Hamdan. "Hair data model: A new data model for Spatio-Temporal data mining." In Data Mining and Optimization, 2012 4th Conference on, pp. 18-22. IEEE, 2012.

[11]. Dabov, Kostadin, et al. "Image denoising with block-matching and 3D filtering." Electronic Imaging 2006. International Society for Optics and Photonics, 2006.

[12]. Yang, Pan, XiaolinGui, Feng Tian, Jing Yao, and Jiancai Lin. "A Privacy-Preserving Data Obfuscation Scheme Used in Data Statistics and Data Mining." In High Performance Computing and Communications \& 2013 IEEE International Conference on Embedded and Ubiquitous Computing, 2013 IEEE 10th International Conference on, pp. 881-887. IEEE, 2013. 